\title{
Quality of Life in Patients with Nasal Obstruction after Septoplasty: A Single Institution Prospective Observational Study
}

\author{
Justyna Dąbrowska-Bieńn ${ }^{1,2}$ Henryk Skarżyński ${ }^{1,2}$ \\ Piotr Henryk Skarżyński ${ }^{1,2,3,4,5}$ \\ ${ }^{1}$ Institute of Physiology and Pathology of Hearing, Warsaw, Poland \\ 2 Otorhinolaryngology Surgery Clinic, World Hearing Center, \\ 3 Department of Heart Failure and Cardiac Rehabilitation, Faculty of \\ Medicine, Medical University of Warsaw, Warsaw, Poland \\ ${ }^{4}$ Department of Teleaudiology and Screening, World Hearing Center, \\ Institute of Physiology and Pathology of Hearing, Kajetany/Warsaw, \\ ${ }^{5}$ Institute of Sensory Organs, Kajetany/Warsaw, Poland
}

\author{
Sebastian Filip Górski ${ }^{1,2}$
}

Int Arch Otorhinolaryngol 2021;25(4):e575-e579.

\author{
Address for correspondence Piotr Henryk Skarzynski, PhD, MSc, MD, \\ World Hearing Center, Kajetany, Poland \\ (e-mail: p.skarzynski@ifps.org.pl).
}

\begin{abstract}
Introduction Nasal obstruction is a common symptom in otorhinolaryngological practice. It can impact significantly on the quality of life of the individual.

Objective The primary goal of the present study was to evaluate quality of life after septoplasty in adults with nasal obstruction. A secondary goal was to assess the effectiveness of septoplasty.

Methods This was a single institution prospective observational study. Patients had experienced septal deviation and symptomatic nasal obstruction with no benefit from medical treatment. There were 51 patients who completed the Nasal Obstruction Symptom Evaluation (NOSE-POL) scale as well as theVisual Analogue Scale (VAS) before undergoing septoplasty, 3 months later, and finally 7 months after surgery. Patients evaluated changes in their nasal obstruction and changes in their quality of life using the Clinical Global Impression Scale (CGI-S).

Results There was a significant improvement in nasal obstruction after septoplasty. Before septoplasty, the mean score on NOSE was 60.3 $\pm 20.4 ; 3$ months after surgery, it was $32.9 \pm 16.8$; and 7 months after surgery it was $39.6 \pm 33.2$. The VAS results also proved a significant enhancement in nasal obstruction after septoplasty. Patients reported an improvement in nasal obstruction as well as a positive change in quality of life, confirming the effectiveness of septoplasty.

Conclusions In patients with deformed septum, septoplasty contributes to high satisfaction of the patient and a compelling improvement in disease-specific quality of life. The NOSE-POL questionnaire is a useful tool for measuring the outcomes of this procedure.
\end{abstract}

\footnotetext{
February 19, 2021

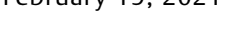

.
}

DOI https://doi.org/ 10.1055/s-0040-1722162. ISSN 1809-9777.

\footnotetext{
(c) 2021. Fundação Otorrinolaringologia. All rights reserved.

This is an open access article published by Thieme under the terms of the Creative Commons Attribution-NonDerivative-NonCommercial-License, permitting copying and reproduction so long as the original work is given
appropriate credit. Contents may not be used for commercial purposes, or appropriate credit. Contents may not be used for commercial purposes, or a dransformed or built upon. (https://creativecommons.org/ licenses/by-nc-nd/4.0/) s.org/ 


\section{Introduction}

Nasal congestion, also called stuffy nose, is a common complaint in otolaryngologist's practice. This problem affects between 9.5 and $15 \%$ of the general population. ${ }^{1}$ In the adult population, it is often a symptom of another health problem, as the main causes for nasal blockage are chronic diseases, such as chronic rhinosinusitis and allergic rhinitis. In Europe, chronic rhinosinusitis has a prevalence of $10.9 \%$, and allergic rhinitis has a prevalence of between 17 and $29 \%{ }^{3}$ The aforementioned diseases cause mucosal congestion, which results in nasal obstruction with decreased nasal airflow. The third main cause for nasal blockage is nasal septal deviation, where abnormalities of the bony and cartilaginous structures of the nose can lead to difficulties in breathing caused by reduced nasal airflow. Deviation of the nasal septum has a prevalence of between 19 and $65 \%$ depending on the criterion for defining a deviated septum. ${ }^{4}$

Not all patients with a deviated septum need surgery to relieve symptoms. In the ear nose and throat (ENT) specialty, nasal septoplasty is the third most common surgery performed. ${ }^{5}$ Several studies have evaluated the outcome of septoplasty, but most of them are retrospective, ${ }^{6}$ conducted on a small group of patients, ${ }^{6}$ include different surgical techniques, ${ }^{5,7,8}$ or are associated with other surgical treatments, ${ }^{9-12}$ which may interfere with their interpretation. There is a poor correlation between objective measurements and subjective nasal potency symptoms: ${ }^{13}$ several studies in which objective measures were used showed no correlation with patient satisfaction after septoplasty. ${ }^{14,15}$ Other studies did not use validated instruments to assess surgical outcomes. ${ }^{5,16}$

Over the last decades, disease-specific instruments that measure nasal obstruction and health-related quality of life have been developed and validated to assess the outcomes of nose surgery. These tools are reliable, reproducible, valid, and sensitive to change. ${ }^{9,17,18}$ We carried the translation and cultural adaptation in Polish of the NOSE questionnaire and confirmed that the Polish version has the same psychometric properties as the original tool. ${ }^{19}$

The primary goal of the present study is to compare the severity of symptoms and the quality of life related to nasal obstruction before septoplasty, 3 months later and, finally, 7 months after surgery. A secondary goal was the assessment of the effectiveness of septoplasty in patients with nasal obstruction.

\section{Method}

\section{Measures}

The participants were asked to complete the Nasal Obstruction Symptom Evaluation (NOSE-POL), the Visual Analogue Scale (VAS), and the Clinical Global Impression Scale (CGI-S).

The NOSE scale is a questionnaire specific to nasal obstruction. It was developed by Stewart et al. in 2004, ${ }^{17}$ and since then has been translated into many languages, adapted, and validated ${ }^{12-17}$. We chose this questionnaire because it is brief, easy for the patient to complete, and specific to nasal obstruction. Furthermore, it can be also applied after septo- plasty, ${ }^{20}$ functional rhinoplasty, ${ }^{9}$ or turbinoplasty. ${ }^{10}$ Patients from our study group completed the NOSE 3 times: once prior to surgery ( 2 weeks before) and then 3 months and 7 months postoperatively.

The visual analogue scale (VAS) was also used. The participants were asked to mark on a horizontal line how difficult it was to breathe through the nose. There was a line $100 \mathrm{~mm}$ long and 3 verbal descriptors (or word anchors): 'none' (on the left); 'medium' (in the center); and 'severe' (on the right). The mark made by the participants was converted into a number from 0 to 10 . The higher the number, the more troublesome was breathing through the nose.

The Clinical Global Impression Scale (CGI-S) is a brief tool used to assess change in a subject's condition. ${ }^{21}$ In our study, the patients were asked to evaluate the change in their nasal obstruction and the change in their quality of life 7 months after septoplasty in comparison with the state before the surgery. The evaluation was done by means of a 7-point scale with the following degrees: 1 , very much worse; 2 , much worse; 3 , minimally worse; 4 , no change; 5 , minimally improved; 6, much improved; and 7, very much improved. Separately, the patients assessed the change in their nasal obstruction and the change in their quality of life, selecting one answer for each.

\section{Subjects}

This was a single institution prospective observational study. The study protocol, informed consent form, and patient information brochure were approved by the Institutional Ethics Committee and accorded with the World Medical Association Declaration of Helsinki. Each patient gave informed written consent for participating in the study.

Initially, the study group consisted of 51 patients. The inclusion criteria were: at least 18 years old; septal deviation consistent with presenting symptom of chronic nasal obstruction; symptoms lasting at least 3 months; and persistent symptoms after a 4-week trial of medical management (including topical nasal steroids, topical or oral decongestants, or an oral antihistamine/decongestant combination). The exclusion criteria were: sinonasal malignancy; radiation therapy to the head or neck; previous surgery (septoplasty, sinus surgery, rhinoplasty, or turbinoplasty); history or clinical evidence of chronic sinusitis (using the criteria from the European Position Paper on Rhinosinusitis and Nasal Polyps 2012)22; adenoid hypertrophy; sleep apnea syndrome; septal perforation; craniofacial syndrome; acute nasal trauma or fracture in the past 3 months; nasal valve collapse; sarcoidosis; Wegener granulomatosis; uncontrolled asthma; pregnancy; or illiteracy.

The patients completed the NOSE-POL 2 weeks before the septoplasty and 3 months later, after surgery, when all patients returned to the outpatient clinic (reporting symptoms at this follow-up should minimize reporting errors). Seven months after the surgery, questionnaires were sent by post to all the patients. The follow-up rate was 53\% - only 27 subjects sent back completed questionnaires. There were 6 women and 21 men aged from 20 to 62 years old (median $[\mathrm{M}]=34.67$; standard deviation $[\mathrm{SD}]=11.95)$. 


\section{Statistical Analysis}

Repeated measures analysis of variance (rANOVA) was conducted to compare baseline and 3-month and 7-month follow-up NOSE-POL scores. Bonferrroni correction was applied for multiple comparisons.

Additionally, pretreatment to post-treatment change in the NOSE-POL and VAS scores was assessed in two other ways. First, with the mean difference between baseline and postoperative results (i.e., the follow-up postoperative score was subtracted from the preoperative score: a positive result indicated improvement (reduction of nasal obstruction symptoms); a negative result indicated deterioration (enhancement of nasal obstruction symptoms). Second, the pretreatment to post-treatment change in the NOSE-POL and VAS scores was assessed as the standardized mean difference and taken as a measure of Cohen effect size ${ }^{23}$ : 0.2 was considered a small effect, 0.5 , a moderate effect, and 0.8 a large effect.

Correlations between change in the NOSE-POL and change in the VAS and changes in nasal obstruction and quality of life were calculated using rho-Spearman correlation. The hypothesis was that the correlations would be positive and at least moderate. The strength of correlation was evaluated according to criteria provided by the British Medical Journal guidelines: ${ }^{24}$ more than 0.8 , a very strong correlation; 0.60.79 , strong; 0.4-0.59, moderate; 0.2-0.39, weak; and below 0.2 , very weak.

A $p$-value $<0.05$ was considered statistically significant. Statistical analysis was conducted using IBM SPSS Statistics for Windows, version 24 (IBM Corp., Armonk, NY).

\section{Results}

\section{Pretreatment and Post-treatment Results}

Baseline NOSE-POL scores (2 weeks before septoplasty) and the results obtained after septoplasty (3 and 7 months postoperatively) are shown in -Table 1.

The results of repeated measures ANOVA showed that the NOSE-POL scores were significantly different: $F=12.62 ; p<$ $0.001 ; e^{2}=0.33$. Post-hoc tests revealed that there was a significant improvement in nasal obstruction 3 months $(p<0.001)$ and 7 months $(p<0.05)$ after septoplasty compared with baseline. A comparison between the 3-month and 7 -month scores was not statistically significant $(p>0.05)$, indicating stability of nasal obstruction symptoms. The mean

Table 1 Scores of the NOSE-POL 2 weeks before septoplasty and 3 months and 7 months after septoplasty

\begin{tabular}{|l|l|l|l|l|l|}
\hline & Min & Max & Me & M & SD \\
\hline Baseline & 15 & 95 & 65 & 60.37 & 20.38 \\
\hline $\begin{array}{l}3 \text { months } \\
\text { postoperatively }\end{array}$ & 0 & 60 & 35 & 32.96 & 16.77 \\
\hline $\begin{array}{l}7 \text { months } \\
\text { postoperatively }\end{array}$ & 0 & 100 & 30 & 39.63 & 33.22 \\
\hline
\end{tabular}

Abbreviations: M, mean; Max, maximum; Me, median; Min, minimum; $\mathrm{SD}$, standard deviation. change in the NOSE-POL scores 3 months after septoplasty was 27.41; $\mathrm{SD}=16.01$ (95\% confidence interval [CI]: 21.133.7) and 7 months after septoplasty it was 20.74; $\mathrm{SD}=37.56$ (95\%CI: 5.9-35.6). The effect size 3 months after the surgery was 1.71 and after 7 months it was 0.55 .

The VAS score before septoplasty was $M=6.33$ $(\mathrm{SD}=2.28)$. A statistically significant improvement in nasal obstruction symptoms was revealed 3 months after septoplasty $(\mathrm{M}=2.39 ; \mathrm{SD}=1.31 ; p<0.001)$, as well as 7 months after surgery $(\mathrm{M}=3.84 ; \mathrm{SD}=3.38 ; p<0.01)$.

The mean change in the VAS score 3 months after septoplasty was $3.94 ; \mathrm{SD}=2.12$ (95\% CI: $3.1-4.8)$ and 7 months after septoplasty it was $2.50 ; \mathrm{SD}=3.54$ (95\%CI: $5.9-35.6$ ). The effect size 3 months after surgery was 1.86 , and 7 months after the surgery it was 0.71 .

\section{Change in the NOSE-POL and Change in Other Measures}

The data in - Table 2 show how the patients assessed the perceived change in their nasal obstruction and the change in their quality of life 7 months after septoplasty.

Twenty-five patients answered the questions concerning change in nasal obstruction and quality of life, and $72 \%$ of them reported improvement in their nasal obstruction 7 months after septoplasty. No change in nasal obstruction was reported by $16 \%$ of the patients and deterioration was reported by $12 \%$.

Some $68 \%$ of the patients declared that their quality of life had improved 7 months after the septoplasty, 20\% reported no change, and $12 \%$ declared that their quality of life had decreased in comparison with the pretreatment period.

For each of the aforementioned mentioned category of patients, the mean change in the NOSE-POLE scores was calculated and is presented in - Fig. $\mathbf{1}$.

It can be seen that the mean change in NOSE-POL scores exhibit an orderly progression from very much or much worse through no change to much or very much improved. The highest change in the NOSE-POL (average 75 points) was in those patients who were the most satisfied with their nasal obstruction according to the CGI-S. Similarly, the patients who reported very much improvement in their quality of life demonstrated a considerable reduction of

Table 2 Subjectively perceived change in nasal obstruction and quality of life 7 months after septoplasty

\begin{tabular}{|l|l|l|}
\hline & $\begin{array}{l}\text { Subjective } \\
\text { change in nasal } \\
\text { obstruction }\end{array}$ & $\begin{array}{l}\text { Subjective } \\
\text { change in } \\
\text { quality of life }\end{array}$ \\
\hline Very much worse & 0 & $1(4 \%)$ \\
\hline Much worse & $3(12 \%)$ & $1(4 \%)$ \\
\hline Minimally worse & 0 & $1(4 \%)$ \\
\hline No change & $4(16 \%)$ & $5(20 \%)$ \\
\hline Minimally improved & $3(12 \%)$ & $4(16 \%)$ \\
\hline Much improved & $12(48 \%)$ & $10(40 \%)$ \\
\hline Very much improved & $3(12 \%)$ & $3(12 \%)$ \\
\hline
\end{tabular}




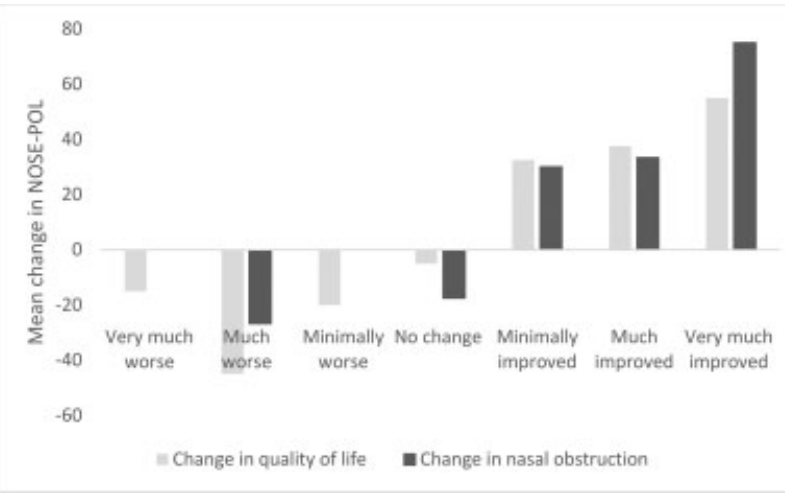

Fig. 1 Mean change in the NOSE-POL score due to change in nasal obstruction and due to change in quality of life 7 months after septoplasty.

complaints in the NOSE-POL. Also, the patients who answered in the CGI-S that their nasal obstruction had worsened had a negative change in the NOSE-POL, indicating deterioration of nasal obstruction symptoms. Thus, the change in the NOSE-POL is consistent with the changes assessed subjectively by other measures, and this is confirmed by the following correlations.

The correlations between change in the NOSE-POL scores and changes assessed with other measures were statistically significant. They were: rho $=0.86 ; p<0.001$ (change in VAS scores), rho $=0.77 ; p<0.001$ (change in nasal obstruction, rho $=0.61 ; p<0.05$ (change in quality of life). All correlations were positive, meaning that the higher the change in NOSE-POL score, the higher the change in the scores of other measures.

\section{Discussion}

Patient-reported outcome measures are widely used among surgical specialties to estimate the impact of interventions on patients' health-related quality of life. There is a wide variety of elective operative procedures in otolaryngology, all of which aim to improve quality of life. As septal deviation has a prevalence of up to $65 \%$, we as doctors should use questionnaires to choose those patients with the greatest likelihood of improvement after septoplasty.

The present research showed a statistically and clinically significant enhancement in quality of life and nasal obstruction scores after septal surgery. The NOSE baseline scores for our patient population $(67.5 \pm 19.5)$ was similar to the study by Stewart et al. ${ }^{9}(60.37 \pm 20.38)$. The NOSE scores after surgery ${ }^{9}$ were also similar ( $26.6 \pm 23.8$ versus $39.63 \pm 33.22)$. We have demonstrated that NOSE scores are dependable on the results from other measures, therefore this self-report questionnaire can be used to assess the effectiveness of septoplasty..

There are controversies in the literature regarding potentially positive results of septoplasty with turbinoplasty versus septoplasty alone. Both Stewart ${ }^{9}$ and Uppal ${ }^{11}$ found no statistically significant difference between either group. Nielsen et al. ${ }^{25}$ compared three groups of patients: septoplasty, radiofrequency therapy of the inferior turbinate
(RFIT), and both procedures. They concluded that patients who underwent RFIT with septoplasty complained less about postoperative nasal congestion than patients who went through RFIT only. We have not performed RFIT on our patients, just septoplasty.

In a systematic review of patient-reported nasal obstruction scores of the PubMed database, ${ }^{20}$ normal and anomalous values of NOSE and VAS scores were settled for clinical use. This approach could be helpful in categorizing the severity of nasal obstruction, guiding treatment, educating patients, and measuring surgical outcomes.

Bugten et al. ${ }^{26}$ revealed that nasal blockage may augment symptoms such as snoring, oral breathing, and nasal discharge, which may therefore weaken the general health of the patient. Surgery leads to a highly significant symptom improvement.

Furthermore, nasal septoplasty is often performed in some patients who have coexisting diseases such as chronic rhinosinusitis, obstructive sleep apnea, asthma, or allergy. These are all conditions that may be affected by nasal blockage. Patients who suffer with allergy might not achieve as good postoperative outcomes as nonallergic patients after surgery. This group should benefit from additional preoperative diagnostic procedures, such as computed tomography (CT) of the nose and sinuses, to improve preoperative planning. Nevertheless, it is strongly recommended that allergic patients also receive medical treatment postoperatively to optimize the results after surgery. ${ }^{26}$

It was shown in the study by Thorstensen ${ }^{27}$ that asthmatic patients have more symptoms of nasal blockage than nonasthmatic patients, and that they need an open nose to optimize airflow to the lower airways. A blocked nose, with consequent lack of humid, warm, and clean inspired air, may harm the lungs and lead to worsening of asthma, so treatment of nasal blockage in asthmatics is particularly important. ${ }^{28}$

Many patients with chronic rhinosinusitis do have a deviated nasal septum, but we excluded this group of patients from our study. The reason was so we could focus solely on nasal obstruction and its impact on quality of life.

The present study has limitations. We have not randomized the patients to other treatment options for comparison. The major strength of the present study is that it is prospective and from one otolaryngology hospital with $>100$ beds. All patients selected for surgery were asked to participate. From the findings of the present study, we encourage other specialists to use the NOSE questionnaire: it can successfully guide treatment and can act as a clinically meaningful measure of surgical outcome.

\section{Conclusions}

Nasal septal surgery leads to a highly significant improvement in disease-specific quality of life. Our patients reported a positive change in nasal obstruction and quality of life after septoplasty.

Trial Registration

April 24, 2016. 


\section{Conflict of interests}

The authors have no conflict to interests to declare.

\section{References}

1 Akerlund A, Millqvist E, Oberg D, Bende M. Prevalence of upper and lower airway symptoms: the Skövde population-based study. Acta Otolaryngol 2006;126(05):483-488

2 Hastan D, Fokkens WJ, Bachert C, et al. Chronic rhinosinusitis in Europe-an underestimated disease. A GA ${ }^{2}$ LEN study. Allergy 2011;66(09):1216-1223

3 Bauchau V, Durham SR. Prevalence and rate of diagnosis of allergic rhinitis in Europe. Eur Respir J 2004;24(05):758-764

4 Smith KD, Edwards PC, Saini TS, Norton NS. The prevalence of concha bullosa and nasal septal deviation and their relationship to maxillary sinusitis by volumetric tomography. Int J Dent 2010; 2010:404982

5 Siegel NS, Gliklich RE, Taghizadeh F, Chang Y. Outcomes of septoplasty. Otolaryngol Head Neck Surg 2000;122(02):228-232

6 Jessen M, Ivarsson A, Malm L. Nasal airway resistance and symptoms after functional septoplasty: comparison of findings at 9 months and 9 years. Clin Otolaryngol Allied Sci 1989;14(03): 231-234

7 Samad I, Stevens HE, Maloney A. The efficacy of nasal septal surgery. J Otolaryngol 1992;21(02):88-91

8 Dinis PB, Haider H. Septoplasty: long-term evaluation of results. Am J Otolaryngol 2002;23(02):85-90

9 Stewart MG, Smith TL, Weaver EM, et al. Outcomes after nasal septoplasty: results from the Nasal Obstruction Septoplasty Effectiveness (NOSE) study. Otolaryngol Head Neck Surg 2004; 130(03):283-290

10 Harrill WC, Pillsbury HC III, McGuirt WF, Stewart MG. Radiofrequency turbinate reduction: a NOSE evaluation. Laryngoscope 2007;117(11):1912-1919

11 Uppal S, Mistry H, Nadig S, Back G, Coatesworth A. Evaluation of patient benefit from nasal septal surgery for nasal obstruction. Auris Nasus Larynx 2005;32(02):129-137

12 Dąbrowska-Bień J, Skarżyński PH, Gwizdalska I, Łazęcka K, Skarżyński H. Complications in septoplasty based on a large group of 5639 patients. Eur Arch Otorhinolaryngol 2018;275(07): 1789-1794

13 André RF, Vuyk HD, Ahmed A, Graamans K, Nolst Trenité GJ. Correlation between subjective and objective evaluation of the nasal airway. A systematic review of the highest level of evidence. Clin Otolaryngol 2009;34(06):518-525
14 Boyce JM, Eccles R. Assessment of subjective scales for selection of patients for nasal septal surgery. Clin Otolaryngol 2006;31(04): 297-302

15 Singh A, Patel N, Kenyon G, Donaldson G. Is there objective evidence that septal surgery improves nasal airflow? J Laryngol Otol 2006;120(11):916-920

16 Arunachalam PS, Kitcher E, Gray J, Wilson JA. Nasal septal surgery: evaluation of symptomatic and general health outcomes. Clin Otolaryngol Allied Sci 2001;26(05):367-370

17 Stewart MG, Witsell DL, Smith TL, Weaver EM, Yueh B, Hannley MT. Development and validation of the Nasal Obstruction Symptom Evaluation (NOSE) scale. Otolaryngol Head Neck Surg 2004; 130(02):157-163

18 Dąbrowska-Bień J, Skarżyński PH. Ocena jakości życia po septoplastykach. 2018;7(01):25-34

19 Dąbrowska-Bień J, Skarżyński H, Gos E, Gwizdalska I, Lazecka KB, Skarżyński PH. Clinical Evaluation of a Polish Translation and Cross-Cultural Adaptation of the Nasal Obstruction Symptom Evaluation (NOSE) Scale. Med Sci Monit 2018;24:7958-7964

20 Rhee JS, Poetker DM, Smith TL, Bustillo A, Burzynski M, Davis RE. Nasal valve surgery improves disease-specific quality of life. Laryngoscope 2005;115(03):437-440

21 Busner J, Targum SD. The clinical global impressions scale: applying a research tool in clinical practice. Psychiatry (Edgmont Pa) 2007;4(07):28-37

22 Fokkens WJ, Lund VJ, Mullol J, et al. European Position Paper on Rhinosinusitis and Nasal Polyps 2012. Rhinol Suppl 2012; 2331-298

23 Cohen J. A power primer. Psychol Bull 1992;112(01):155-159

24 Correlation and regression | The BMJ. https://www.bmj.com/about$\mathrm{bmj} /$ resources-readers/publications/statistics-square-one/ 11-correlation-and-regression. Accessed December 15, 2019.

25 Nilsen AH, Helvik A-S, Thorstensen WM, Bugten V. A comparison of symptoms and quality of life before and after nasal septoplasty and radiofrequency therapy of the inferior turbinate. BMC Ear Nose Throat Disord 2018;18:2

26 Bugten V, Nilsen AH, Thorstensen WM, Moxness MHS, Amundsen MF, Nordgård S. Quality of life and symptoms before and after nasal septoplasty compared with healthy individuals. BMC Ear Nose Throat Disord 2016;16:13

27 Thorstensen WM, Bugten V, Sue-Chu M, Fossland NPW, Romundstad PR, Steinsvåg SK. Sino-nasal characteristics in asthmatic patients. Otolaryngol Head Neck Surg 2012;147(05):950-957

28 Randolph CC. Allergic rhinitis and asthma in the athlete. Allergy Asthma Proc 2006;27(02):104-109 